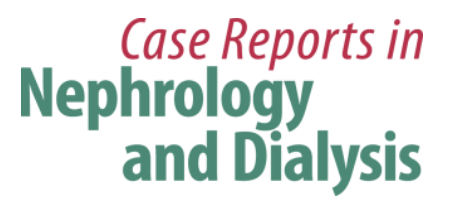

Case Rep Nephrol Dial 2017;7:81-90

DOI: $10.1159 / 000477660$

Published online: June 23, 2017

(c) 2017 The Author(s)

Published by S. Karger AG, Basel

www.karger.com/cnd

This article is licensed under the Creative Commons Attribution-NonCommercial 4.0 International License (CC BY-NC) (http://www.karger.com/Services/OpenAccessLicense). Usage and distribution for commercial purposes requires written permission.

\title{
Case Report of Spontaneous Remission of Biopsy-Proven Idiopathic Immune Complex-Mediated Membranoproliferative Glomerulonephritis
}

\author{
Rehan Shah $^{\text {a }}$ Mark S. Segal ${ }^{b} \quad$ Michael J. Wilkowski ${ }^{c}$ \\ ${ }^{a}$ Tift Regional Medical Center, Tifton, GA, USA; ${ }^{b}$ Division of Nephrology, University of \\ Florida School of Medicine, Gainesville, FL, USA; ${ }^{C}$ Mercer University School of Medicine - \\ Savannah Campus, Savannah, GA, USA
}

\section{Keywords}

Immune complex · Membranoproliferative glomerulonephritis · Therapy

\begin{abstract}
Membranoproliferative glomerulonephritis (MPGN) is a histopathologic diagnosis causing microscopic hematuria, nephrotic range proteinuria, and chronic renal failure. Current understanding divides pathogenesis into two broad categories: immune complex mediated and complement mediated (now termed C3 glomerulopathy). The term idiopathic immune complex-mediated MPGN would apply to a patient without an identifiable source of immune complex production and no evidence of C3 glomerulopathy. Presented is a patient with idiopathic immune complex mediated MPGN and her clinical course. The patient opted for conservative therapy with losartan, carvedilol, chlorthalidone, and atorvastatin. Nephrotic range proteinuria of $8.7 \mathrm{~g}$ per day resolved over 5 months, with improvement of serum from 3.3 to $1.2 \mathrm{mg} / \mathrm{dL}$. Remission continues at follow-up 21 months after biopsy. For idiopathic immune
\end{abstract}




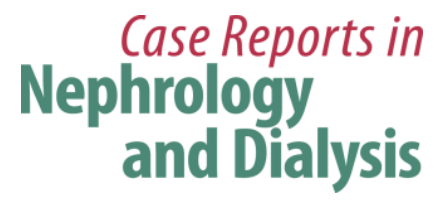

Case Rep Nephrol Dial 2017;7:81-90

(C) 2017 The Author(s). Published by S. Karger AG, Basel www.karger.com/cnd

Shah et al:: Case Report of Spontaneous Remission of Biopsy-Proven Idiopathic Immune Complex-Mediated Membranoproliferative Glomerulonephritis

complex-mediated MPGN, resorting to empiric immunosuppression therapy may not be the best option. As this patient demonstrates, a conservative approach of blood pressure control with anti-renin-angiotensin agents, control of lipids, and watchful follow-up can be successful.

(C) 2017 The Author(s)

Published by S. Karger AG, Basel

\section{Introduction}

The histopathologic diagnosis of membranoproliferative glomerulonephritis (MPGN) is clinically marked by microscopic hematuria, nephrotic range proteinuria, and chronic renal failure. The natural history is usually one of progressing to end-stage kidney disease. A recent revision of the clinical classification divides MPGN based on immunofluorescence into two categories: (1) immune complex-mediated and (2) complement-mediated diseases [1]. The immune complex-mediated group can be further subdivided by the cause of the immune complex formation, yielding 3 general sources including infection related (most commonly hepatitis $\mathrm{C}$ ), monoclonal protein deposition, or an associated autoimmune disease such as systemic lupus erythematosus.

The term idiopathic immune complex-mediated MPGN refers to the rare occasion where there is no evidence of infection, no monoclonal protein deposition, and no identifiable systemic autoimmune disease [2]. In such patients, the question arises as to whether to add immunosuppression empirically to the usual care of chronic glomerulonephritis, reninangiotensin-aldosterone system (RAAS) inhibition and careful control of hypertension and hyperlipidemia. We present a patient with biopsy-proven idiopathic immune complexmediated MPGN who responded predominantly with RAAS inhibition, blood pressure control, and statin therapy with improvement in renal function and remission of nephrotic range proteinuria over a period of 6 months and sustained remission at 18 months. In this case, the patient's immediate treatment with conservative therapy and watchful waiting spared her prolonged immunosuppression and possible adverse drug effects.

\section{Case Report}

The patient is a 53-year-old woman, a registered nurse, who presented to the emergency department with a chief complaint of weakness, headache, and blurry vision. She reported a 15-pound weight loss over the past 4 months, which she attributed to nausea, anorexia, and intermittent vomiting. Serum chemistries revealed blood urea nitrogen (BUN) of 36 $\mathrm{mg} / \mathrm{dL}$ and serum creatinine of $3.3 \mathrm{mg} / \mathrm{dL}$. Electrolytes were normal as were her liver function tests. Complete blood count was normal and eosinophilia was absent. Initial urinalysis contained 3+ blood, 4+ protein, $32 \mathrm{RBC} / \mathrm{HPF}, 7 \mathrm{WBC} / \mathrm{HPF}$, numerous hyaline casts, and no red blood cell casts. Renal ultrasonography demonstrated normal-size kidneys with increased echogenicity and no hydronephrosis.

Twelve months prior to admission, chemistries revealed a BUN of $25 \mathrm{mg} / \mathrm{dL}$ and serum creatinine of $0.9 \mathrm{mg} / \mathrm{dL}$, and 18 months prior to admission, BUN was $23 \mathrm{mg} / \mathrm{dL}$ with a serum creatinine of $1.0 \mathrm{mg} / \mathrm{dL}$. 


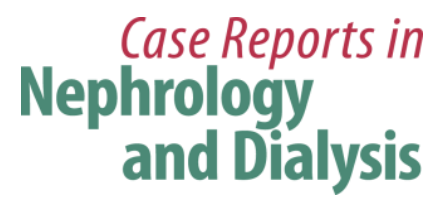

Case Rep Nephrol Dial 2017;7:81-90

DOI: $10.1159 / 00047766$

(c) 2017 The Author(s). Published by S. Karger AG, Basel www.karger.com/cnd

Shah et al.: Case Report of Spontaneous Remission of Biopsy-Proven Idiopathic Immune Complex-Mediated Membranoproliferative Glomerulonephritis

Her past medical history included hypertension treated for the past 4 years with bisoprolol plus hydrochlorothiazide (Ziac). She had a history of pseudotumor cerebri treated with acetazolamide (Diamox). She was diagnosed with hyperlipidemia, but was not on treatment. Four years prior to admission, she underwent laparoscopic hysterectomy and sacrocolpopexy and colpoperineorrhaphy and cystoscopy. The surgery included use of Restorelle ${ }^{\mathrm{TM}}$ mesh and repair of a rectocele and enterocele. As a young adult, she underwent tonsillectomy and adenoidectomy and was involved in a motor vehicle accident with fracture of her left leg. The patient's maternal grandfather had diabetes, both parents have hypertension, and her one adult daughter is alive and well. She has no siblings. There was no history of autoimmune disease, glomerulonephritis, or chronic renal failure among her relatives. The patient had no history of international travel.

Review of system was negative for fever, chills, or night sweats. She experienced occasional palpitations and nonexertional chest pain. She described the chest pain as "acid reflux" and wondered if this caused her nausea and vomiting as well. She denied diarrhea, melena, and hematemesis. She noted trace edema and occasional foamy urine on and off for the past 6 months but no flank pain, dysuria, or visible hematuria. She denied neurologic symptoms, specifically no headache, vision changes, ataxia, or incontinence. She denied dry eyes, dry mouth, joint swelling, skin rashes, sun sensitivity, easy bruising, pleuritic pain, cough, or hemoptysis.

On physical examination, she was a well-appearing woman of Anglo-European heritage. Neurologic examination was normal. Cardiac examination revealed normal S1 and S2 and no murmur. Pulmonary examination showed clear lungs. Abdomen was benign without guarding and no bruits. No lymphadenopathy was present. She had no joint effusions or signs of arthritis. Lower extremities edema was only 1+ bilaterally.

During her hospital course, her bisoprolol and hydrochlorothiazide were discontinued and her hypertension was treated with carvedilol and amlodipine. She received intravenous fluids, but serum creatinine did not change. A 24-h urine collection contained a total volume of 1,450 mL, a total urine protein of $8,764 \mathrm{mg}$ per $24 \mathrm{~h}$, and total urine creatinine of 1,145 mg. Urine creatinine concentration was $79 \mathrm{mg} / \mathrm{dL}$, with a serum creatinine of $3.5 \mathrm{mg} / \mathrm{dL}$; thus, measured creatinine clearance was $22 \mathrm{~mL} / \mathrm{min}$.

A renal biopsy was performed and revealed a MPGN pattern. Double contours and suspected subendothelial deposits were appreciated on light microscopy. No thrombi, necrosis, or crescents were present. There was mild diffuse mononuclear cell interstitial infiltration and tubulitis noted. The interstitium had mild fibrosis. There was mild intimal thickening of the small arteries. Electron microscopy confirmed electron-dense subendothelial deposits without substructure. Since no glomeruli were present in the specimen submitted for immunofluorescence, a pronase antigen retrieval technique was used on sections prepared from the paraffin block. It revealed granular capillary wall staining with 2-3+ IgG, 2-3+ C1q, and $2-3+$ kappa light chain and 1-2+ lambda light chain positivity. There was trace IgM and no IgA staining (Fig. 1a-c).

The patient was discharged home on carvedilol and lisinopril in lieu of the amlodipine. Before she was able to return to the renal clinic, she was readmitted 1 week after discharge for intractable nausea and vomiting. Admission blood pressure was 206/110. This was attributed to her inability to take her antihypertensive medications. Despite this, her serum creatinine improved to $3.1 \mathrm{mg} / \mathrm{dL}$ with BUN $22 \mathrm{mg} / \mathrm{dL}$. Albumin level remained $3.4 \mathrm{~g} / \mathrm{dL}$. 


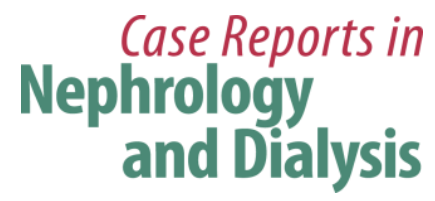

Case Rep Nephrol Dial 2017;7:81-90

DOI: $10.1159 / 00047766$

C 2017 The Author(s). Published by S. Karger AG, Basel www.karger.com/cnd

Shah et al:: Case Report of Spontaneous Remission of Biopsy-Proven Idiopathic Immune Complex-Mediated Membranoproliferative Glomerulonephritis

Urinalysis documented $3+$ blood and $4+$ protein as on her prior admission with numerous hyaline and granular casts per low power field. A 24-h urine collection contained a total volume of $875 \mathrm{~mL}$ and total urine creatinine of $744 \mathrm{mg}$. The urine creatinine concentration was $85 \mathrm{mg} / \mathrm{dL}$. Total urine protein was 4,148 mg per $24 \mathrm{~h}$. At the time of the 24 -h collection, her serum creatinine was $3.7 \mathrm{mg} / \mathrm{dL}$ and creatinine clearance was $18 \mathrm{~mL} / \mathrm{min}$. Upper endoscopy demonstrated gastritis. Biopsies of the esophagus and duodenum were normal. Helicobacter pylori testing was negative. She was treated with an aggressive regimen of sucralfate, proton pump inhibitor, and $\mathrm{H} 2$ blocker.

Upon return to the clinic, 1 month after the biopsy, her blood pressure was reasonably controlled on lisinopril and carvedilol. Bone density was obtained and was normal. 25hydroxyvitamin D was low at $19 \mathrm{ng} / \mathrm{mL}$ and ergocalciferol was started. By this time, a serologic search for an associated systemic disease included negative ANA, anti-double stranded DNA, HIV 1-2, anti-GBM, anti-neutrophil cytoplasmic antibodies (ELISA), and rheumatoid factor. The sedimentation rate was elevated at $75 \mathrm{~mm} / \mathrm{h}$, and repeat measurement was $111 \mathrm{~mm} / \mathrm{h}$. Complement levels were unremarkable with C3 $120 \mathrm{mg} / \mathrm{dL}$ and C4 slightly high at $41 \mathrm{mg} / \mathrm{dL}$. Serum protein immunoelectrophoresis demonstrated normal IgA, low IgG (287 mg/dL), low IgM (38 mg/dL), and no monoclonal spike. Urine protein immunoelectrophoresis was without monoclonal spike, and the kappa/lambda light chain ratio was 3.28 (normal 2.04-10.37). Hemoglobin A1c level returned at 5.8\%. Treatment with antacids, Lisinopril, and carvedilol was continued, and she was instructed to start prednisone $60 \mathrm{mg}$ daily.

Eight weeks after the biopsy, the patient was evaluated at a tertiary care center. She reported taking 3 doses of prednisone but stopped it completely after an episode of visible hematuria. Lisinopril was changed to irbesartan due to persistent dry cough. Laboratory values at this time included a 24-h urine collection with a total volume of $950 \mathrm{~mL}$, total urine creatinine of $950 \mathrm{mg}$, and urine creatinine concentration of $100 \mathrm{mg} / \mathrm{dL}$. Total urine protein was $2,812 \mathrm{mg}$ per $24 \mathrm{~h}$. A separate random urine sample for protein to creatinine ratio was 1.67 (239/143). Anti-scl-70 was negative. She was counseled to not restart prednisone.

By week 12, her blood pressure was moderately controlled at 150/90. Because of cost, irbesartan was discontinued and losartan $100 \mathrm{mg}$ daily was started. Chlorthalidone $25 \mathrm{mg}$ daily was added for her blood pressure control. Atorvastatin $40 \mathrm{mg}$ once a day was started for hyperlipidemia. She continued on carvedilol and antacids. Examination revealed no edema.

At her tertiary clinic visit 44 weeks after the biopsy, she reported feeling well and examination revealed blood pressure of $106 / 70$ and no edema. Laboratory testing in the clinic included serum creatinine $1.6 \mathrm{mg} / \mathrm{dL}$, BUN $25 \mathrm{mg} / \mathrm{dL}$, serum albumin $4.5 \mathrm{~g} / \mathrm{dL}$, and a protein to creatinine ratio of 0.06 . Urinalysis registered trace blood and no protein. One week prior to this visit, micro-albumin assay contained $7.4 \mathrm{mg} / \mathrm{g}$ (normal $<15 \mathrm{mg} / \mathrm{g}$ ), and the protein to creatinine ratio was 0.06 .

Tertiary clinic visit 82 weeks after the biopsy demonstrated well-controlled blood pressure of $117 / 85$. Urinalysis was negative for blood, protein, cells, and casts. Remission was sustained at 92 weeks with stable serum creatinine at $1.2 \mathrm{mg} / \mathrm{dL}$, normal serum albumin, and inactive urinalysis without proteinuria.

Her serial serum creatinine, serum albumin, and urine protein to creatinine ratio values are listed in Table 1. 


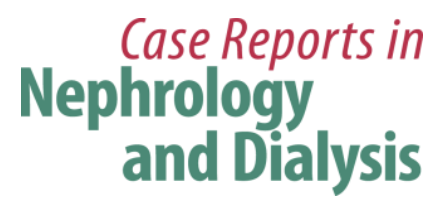

Case Rep Nephrol Dial 2017;7:81-90

DOI: $10.1159 / 00047766$

(c) 2017 The Author(s). Published by S. Karger AG, Basel www.karger.com/cnd

Shah et al.: Case Report of Spontaneous Remission of Biopsy-Proven Idiopathic Immune Complex-Mediated Membranoproliferative Glomerulonephritis

\section{Discussion}

This 53-year-old woman presented with symptoms and signs of malignant hypertension, presumably due to her renal parenchymal disease that she acquired within the past year, along with the findings of advanced renal insufficiency and nephrotic range proteinuria. Biopsy revealed features of MPGN with typical mesangial and endocapillary hypercellularity and double contours evident on light and electron microscopy. Electron microscopy disclosed subendothelial deposits composed of, as immunofluorescence demonstrated, IgG and C1q. No intramembranous deposits were present, bright C3 staining was absent, and immunofluorescence microscopy revealed staining for both kappa and lambda light chains.

For clinical decision-making, classification of MPGN based on pathogenesis is most helpful and the best guide for therapy. Clinical classification divides MPGN into two categories: (1) immune complex-mediated and (2) complement-mediated diseases [1]. C3 glomerulopathy, with characteristic bright C3 staining without substantial immunoglobulin deposition, is an example of the complement-mediated form of MPGN. Dense deposit disease with extensive osmiophilic intramembranous sausage-shaped deposits with immunoglobulin staining is attributed to dysregulation of the complement cascade and is in the same spectrum with C3 glomerulopathy [3]. Recognition of these complement-mediated diseases must be identified by the nephrologist and nephropathologist when MPGN is encountered on renal biopsy since monoclonal antibody therapy against the terminal products of the complement cascade, eculizumab, has been reported to help these patients [4-6].

For MPGN with immune complex deposition, treatment targets the source of immune complex production. The three general sources of pathologic immunoglobulin and immune complexes are infection, monoclonal gammopathy-associated diseases, and autoimmune disorders.

With the identification of hepatitis $\mathrm{C}$, infection is the leading infectious cause of MPGN. Response to antiviral therapy can mitigate the course of the renal disease [7]. A caveat is the occurrence of rapidly progressive glomerulonephritis caused by hepatitis C-associated cryoglobulinemia which requires intense immunosuppression with pulse solumedrol followed by high-dose daily steroids, cyclophosphamide, therapeutic apheresis and the consideration of anti-B-cell therapy in the form of rituximab [8].

Immune complex deposition formed by monoclonal gammopathy from B-cell dyscrasias responds to immunosuppressive treatment. If criteria are met for multiple myeloma, treatment of the primary disease is indicated, though overt myeloma is usually not the culprit when MGPN with IgG monoclonal deposits is the diagnosis [9]. If there is evidence of cryoglobulin production and deposition on renal biopsy, then high-dose daily steroids plus cyclophosphamide is indicated. Therapeutic apheresis would be added for rapidly progressive disease as well as the consideration of anti-B-cell therapy in the form of rituximab. A recent case series of proliferative glomerulonephritis with C3 deposition and monoclonal gammopathy indicated a possible role of the monoclonal protein causing disruption of complement regulation. Treatment of an underlying B-cell dyscrasia improved hematuria and proteinuria and stabilized renal function in this series of patients [10].

The third source of immune complex-mediated MPGN is a systemic autoimmune disease. Systemic lupus erythematosus is the most commonly recognized autoimmune cause, 


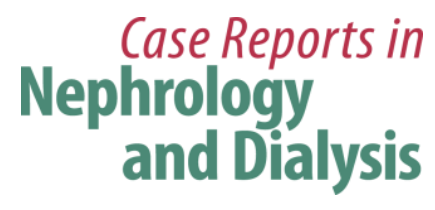

Case Rep Nephrol Dial 2017;7:81-90

DOI: $10.1159 / 00047766$

(C) 2017 The Author(s). Published by S. Karger AG, Base www.karger.com/cnd

Shah et al:: Case Report of Spontaneous Remission of Biopsy-Proven Idiopathic Immune Complex-Mediated Membranoproliferative Glomerulonephritis

and an association with rheumatoid arthritis and Sjögren syndrome has been noted [1]. In this patient, anti-SSA and anti-SSB was not measured; however, the lack of xerophthalmia and xerostomia, negative rheumatoid factor and ANA, low immunoglobulin levels, and the lack of evidence of cryoglobulin deposition make Sjögren syndrome unlikely. As in our patient, remission without immunosuppression of MPGN associated with Sjögren syndrome has been reported [11].

In the present case of MPGN, evidence for complement-mediated disease was absent. Immune complex formation rules out an episode of malignant hypertension as the primary cause in this patient even though her blood pressure was markedly elevated early in her course. Working through the differential for a source of the immune complex formation, there was no evidence of infection or monoclonal protein production or deposition; and autoimmune disease is a possible etiology with extensive subendothelial deposits with multiple immune reactants (IgG, C1q), but unlikely given no apparent disease based on history, physical examination, or serologic testing. Thus, we believe, idiopathic immune complexmediated MPGN is the most accurate diagnosis [2].

At presentation, the patient's clinical prognostic risk factors were not in her favor. Nephrotic syndrome and interstitial fibrosis, which this patient demonstrated, predicts progression in MPGN [12]. Presence of crescents, which this patient did not have, also predicts progression to end-stage disease [12]. Nephrotic range proteinuria along with uncontrolled hypertension at the time of presentation correlate with progression to end-stage disease in patients with proteinuric nephropathies [13]. Her multiple risk factors for accelerated progression make treatment with immunosuppression reasonable [14]. On the other hand, a conservative approach could be supported by the fact that serum albumin at the time of presentation was near normal. In addition, after a short period of observation (2-4 weeks), there was improvement in proteinuria and serum creatinine. Indeed, conservative therapy with renin-angiotensin-aldosterone system inhibition, statin therapy, and blood pressure control, though her blood pressure was not consistently at goal, was enough to improve proteinuria. Although she was briefly exposed to prednisone $60 \mathrm{mg}$ daily, a quantitated total urine protein was on the decline prior to prednisone treatment and it followed a linear path without apparent influence by the few days of prednisone therapy. The gratifying improvement in renal function may be attributed to the absence of an identifiable source of ongoing immune complex production, i.e., the immune-mediated injury was self-limited, allowing for repair without fibrosis. Another speculative explanation for creatinine improvement may be the possible presence of malignant hypertension at the time of admission which resolved with reasonable blood pressure control. However, on renal histopathology, only mild arterial intimal thickening was noted without severe musculomucoid intimal hyperplasia, arguing against an element of malignant hypertension.

If her nephrotic range proteinuria relapses or her glomerular filtration rate declines, another search for systemic autoimmune disease would be warranted. Re-biopsy would also have to be considered. Empiric therapy with rituximab would be in the armamentarium [15]. Although experience with specific class of glomerulonephritis is limited, rituximab has shown benefit in other idiopathic immune complex-mediated glomerular diseases such as membranous glomerulopathy and in nonimmune complex-mediated idiopathic disease such as minimal change disease and focal segmental glomerulosclerosis. Rituximab also has a role in achieving remission or treating relapse in antineutrophil cytoplasmic antibody-associated 


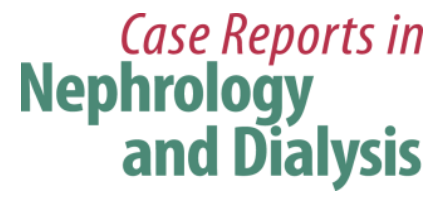

Case Rep Nephrol Dial 2017;7:81-90

DOI: $10.1159 / 00047766$

(C) 2017 The Author(s). Published by S. Karger AG, Basel www.karger.com/cnd

Shah et al:: Case Report of Spontaneous Remission of Biopsy-Proven Idiopathic Immune Complex-Mediated Membranoproliferative Glomerulonephritis

glomerulonephritis and non-cryoglobulinemic monoclonal IgG deposition disease. Without evidence for C3 nephropathy or dense deposit disease, monoclonal antibody therapy with eculizumab is not indicated.

Thus, histological features summarized for a precise pathologic diagnosis, classification based on pathogenesis, and complete clinical evaluation and careful follow-up are the key factors to management of a patient with idiopathic immune complex-mediated MPGN. Our patient's clinical course on conservative therapy has been favorable with remission of proteinuria and more than doubling of her glomerular filtration rate compared to the time of presentation, suggesting that immunosuppression therapy may not be necessary in all cases of idiopathic MPGN.

\section{Statement of Ethics}

Written informed consent was obtained from the patient for publication of this case report and images.

\section{Disclosure Statement}

The authors declare that they have no competing interests.

\section{References}

1 Sethi S, Fervenza FC: Membranoproliferative glomerulonephritis - a new look at an old entity. N Engl J Med 2012;366:1119-1131.

2 Fervenza FC, Sethi S, Glassock RJ: Idiopathic membranoproliferative glomerulonephritis: does it exist? Nephrol Dial Transplant 2012;27:4288-4294.

-3 Sethi S, Fervenza FC, Zhang Y, et al: Proliferative glomerulonephritis secondary to dysfunctiontion of the alternative pathway of complement. Clin J Am Soc Nephrol 2011;6:1009-1012.

-4 Radhakrishnan S, Lunn A, Kirschfink M, et al: Eculizamab and refractory membranoproliferative glomerulonephritis. N Engl J Med 2012;366:1165-1176.

Bomback AS, Smith RJ, Barile GR, et al: Eculizamab for dense deposit disease and C3 glomerulonephritis. Clin J Am Soc Nephrol 2012;7:748-756.

6 Le Quintrec M, Lionet A, Kandel C, et al: Eculizamab for treatment of rapidly progressive C3 glomerulopathy. Am J Kidney Dis 2015;65:484-489.

7 Johnson RJ, Gretch DR, Yamabe H, et al: Membranoproliferative glomerulonephritis associated with hepatitis C infection. N Engl J Med 1993;328:465-470.

8 Dammacco F, Sansonno D: Therapy for hepatitis C virus-related cryoglobulinemic vasculitis. N Engl J Med 2013;369:1035-1045.

-9 Nasr SH, Satoskar A, Markowitz GS, Valeri AM: Proliferative glomerulonephritis with monoclonal IgG deposits. J Am Soc Nephrol 2009;20:2055-2064.

10 Zand L, Kattah A, Fervenza FC, et al: C3 glomerulonephritis associated with monoclonal gammopathy. Am J Kidney Dis 2013;62:506-514.

11 Cortez MS, Sturgill BC, Bolton WK: Membranoproliferative glomerulonephritis with primary Sjogren's syndrome. Am J Kidney Dis 1995;25:632-636.

12 Little MA, Dupont P, Campbell E, et al: Severity of primary MPGN type, determines renal survival and post-tranplantation recurrence risk. Kidney Int 2006;69:504-511. 
13 Ruggenenti P, Perna A, Mosconi L, Pisoni R, Remuzzi G: Urinary protein excretion rate is the best independent predictor of ESRF in non-diabetic proteinuric chronic nephropathies. Kidney Int 1998;53:1209-1216.

14 Fervenza FC, Sethi S: Evaluation and treatment of membranoproliferative glomerulonephritis; in Glassock RJ (ed): UpToDate, Waltham, MA (accessed on December 11, 2016).

15 Dillon JJ, Hladunewich M, Haley WE, Reich HN: Rituximab therapy for type I membranoproliferative glomerulonephritis. Clin Nephrol 2012;77:290-295. 


\section{Case Reports in Nephrology and Dialysis}

Case Rep Nephrol Dial 2017;7:81-90

(C) 2017 The Author(s). Published by S. Karger AG, Basel www.karger.com/cnd

Shah et al: Case Report of Spontaneous Remission of Biopsy-Proven Idiopathic Immune Complex-Mediated Membranoproliferative Glomerulonephritis
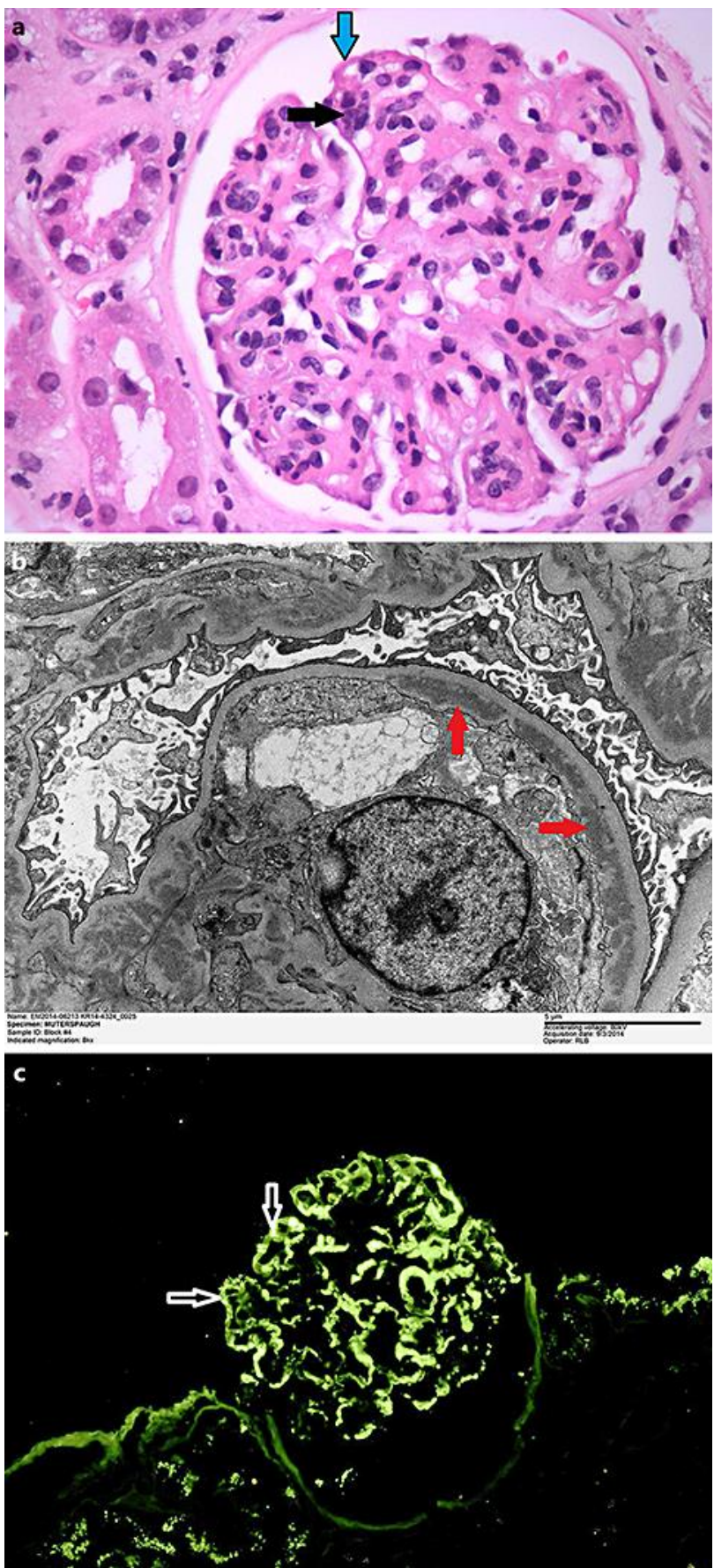

Fig. 1. a Light microscopy of a representative glomerulus revealing a membranoproliferative glomerulonephritis pattern with mesangial proliferation (black arrow) and thickened capillary walls (blue arrow). b Electron microscopy confirmed electron-dense subendothelial deposits without substructure (red arrows). c Immunofluorescence microscopy demonstrating capillary wall 2-3+ IgG staining in a granular pattern (white arrows) and global distribution. 
Shah et al.: Case Report of Spontaneous Remission of Biopsy-Proven Idiopathic Immune Complex-Mediated Membranoproliferative Glomerulonephritis

Table 1. Serial serum creatinine, serum albumin, and urine protein to creatinine ratio values of the patient

\begin{tabular}{llll}
\hline & $\begin{array}{l}\text { Serum } \\
\text { creatinine, } \\
\text { mg/dL }\end{array}$ & $\begin{array}{l}\text { Blood urea } \\
\text { nitrogen, } \\
\text { mg/dL }\end{array}$ & $\begin{array}{l}\text { Protein/ } \\
\text { creatinine } \\
\text { ratio }\end{array}$ \\
\hline Admission & 3.3 & 36 & 7.65 \\
2 weeks after the biopsy & 3.1 & 20 & 5.9 \\
8 weeks after the biopsy & 2.9 & 19 & 1.67 \\
12 weeks after the biopsy & 2.3 & 32 & 2.28 \\
20 weeks after the biopsy & 2.1 & 31 & \\
44 weeks after the biopsy & 1.6 & 25 & 0.06 \\
70 weeks after the biopsy & 1.4 & 27 & 0.06 \\
92 weeks after the biopsy & 1.2 & 23 & \\
\hline
\end{tabular}

